\title{
Coexistence of Lung Squamous Cell Carcinoma and Pulmonary Tuberculosis Within a Single Lesion: A Case Report
}

This article was published in the following Dove Press journal: OncoTargets and Therapy

Ming-Jing $\mathrm{Yu}^{\mathrm{l}, *}$

Pei-Jun $\mathrm{Li}^{2}$

Zong-An Liang (D) ${ }^{2}$

'West China School of Medicine, Sichuan University, Chengdu, Sichuan, People's Republic of China; ${ }^{2}$ Department of Respiratory and Critical Care Medicine, West China Hospital, Sichuan University, Chengdu, Sichuan, People's Republic of China

*These authors contributed equally to this work

\begin{abstract}
Pulmonary tuberculosis (TB) and lung cancer are both common diseases with poor prognosis and high mortality worldwide. The coexistence of the two diseases has rarely been reported while their relationship has been noted. Here we describe a patient diagnosed with both TB and squamous cell carcinoma in a single lesion. The patient had a cough for four months and polypnea for two months, with a smoking history of over 40 years. Chest computed tomography (CT) showed a lobular mass in the right hilar region, which was diagnosed as TB by transbronchial lung biopsy. The symptoms and CT findings indicated the possibility of lung cancer. So, the patient underwent a further lung biopsy at the periphery of the mass, which was confirmed as squamous cell carcinoma. This case illuminated that when the mass with cancer-like morphologic features and location instead of typical TB, even the initial pathological result shows TB, coexistence of the diseases should be considered.
\end{abstract}

Keywords: pulmonary tuberculosis, squamous cell carcinoma, case report

\section{Introduction}

TB is a main cause of death especially in developing countries, which is an important public health issue. Lung cancer is one of the leading causes of cancerrelated death worldwide. Squamous cell carcinoma, related to cigarette smoking, is one of the subtypes of lung cancer. ${ }^{1}$ The coexistence of pulmonary TB and lung cancer in different lobes has been reported in clinical observation studies, but the two diseases occurring simultaneously in same lesion are rare. ${ }^{2-4}$ Here, we report a case with imaging findings of lung cancer but diagnosed as pulmonary TB by transbronchial lung biopsy (TBLB). Further biopsy showed the coexistence of pulmonary TB and lung cancer in the same upper right lobe lesion.

\section{Case Description}

A 53-year-old man presented to our hospital with a 4-month history of cough and polypnea for 2 months. He was a former smoker and consumed 30 cigarettes daily for 40 years, and no particular personal or family medical history was reported. Physical examination showed stable vital signs, including blood pressure of 126/86 $\mathrm{mmHg}$, pulse rate of 92 beats/min, respiratory rate of $20 / \mathrm{min}$, and temperature of $36.4{ }^{\circ} \mathrm{C}$. Weakened breathing sounds were detected in the right lung. Cytokeratin fragment (CYFRA) 21-1 was elevated to $4.62 \mathrm{ng} / \mathrm{mL}$, while other laboratory parameters were unremarkable. Bacteriology of sputum was negative for tubercle bacilli. The chest CT
Correspondence: Zong-An Liang

Email liangza@scu.edu.cn 
at admission showed a $5.9 \mathrm{~cm} \times 3.9 \mathrm{~cm}$-sized lobular mass in the right hilar region with an irregular margin. The contrastenhanced CT demonstrated heterogeneous enhancement of the mass, without necrosis or cavity, but with a contiguous tracheobronchial stenosis in the right upper-middle lobe. Adenopathy in the right hilar and mediastinum region was observed. Lesions did not demonstrate any cavitary, fiber, strips, and calcified shadow changes (Figure 1). Besides, distant metastasis was not found by an enhanced head magnetic resonance imaging, an enhanced abdominal CT and a full-body bone scan.

The patient underwent the TBLB in the hilar region, which showed that the mucosa in the right main bronchus swelled, and the right upper bronchial linear narrowed. When the tracheoscopy passed through the right middle bronchus, the mass in the hilar region was seen to invading the carina tracheal tube and easily to bleed when touched. Pathologic results by the TBLB revealed the mass in the right hilar region as chronic inflammation with necrosis, and the DNA fragment of Mycobacterium tuberculosis (Mtb) was found in the specimen (Figure 2). Considering that the patient had no history of tuberculosis and the CT scan showed no features of obsolete tuberculosis, the patient was diagnosed with TB. However, the CT and the TBLB findings still indicated highly possibility of coexisting lung cancer. Thus, the patient received a CT-guided percutaneous lung biopsy at the periphery of the same mass (Figure 3). The pathology showed a poorly differentiated squamous cell carcinoma (Figure 2).

Thus, the final main diagnosis was squamous cell lung cancer (cT4N2M0, stage IIIB). The second diagnosis was pulmonary TB. The patient was then suggested to start anti-TB treatment and assess chemotherapy with a readmission after anti-TB therapy for more than two weeks. Meanwhile, genetic testing for lung cancer was also recommended to conduct for the further evaluation of possible targeted drug therapy. Unfortunately, the
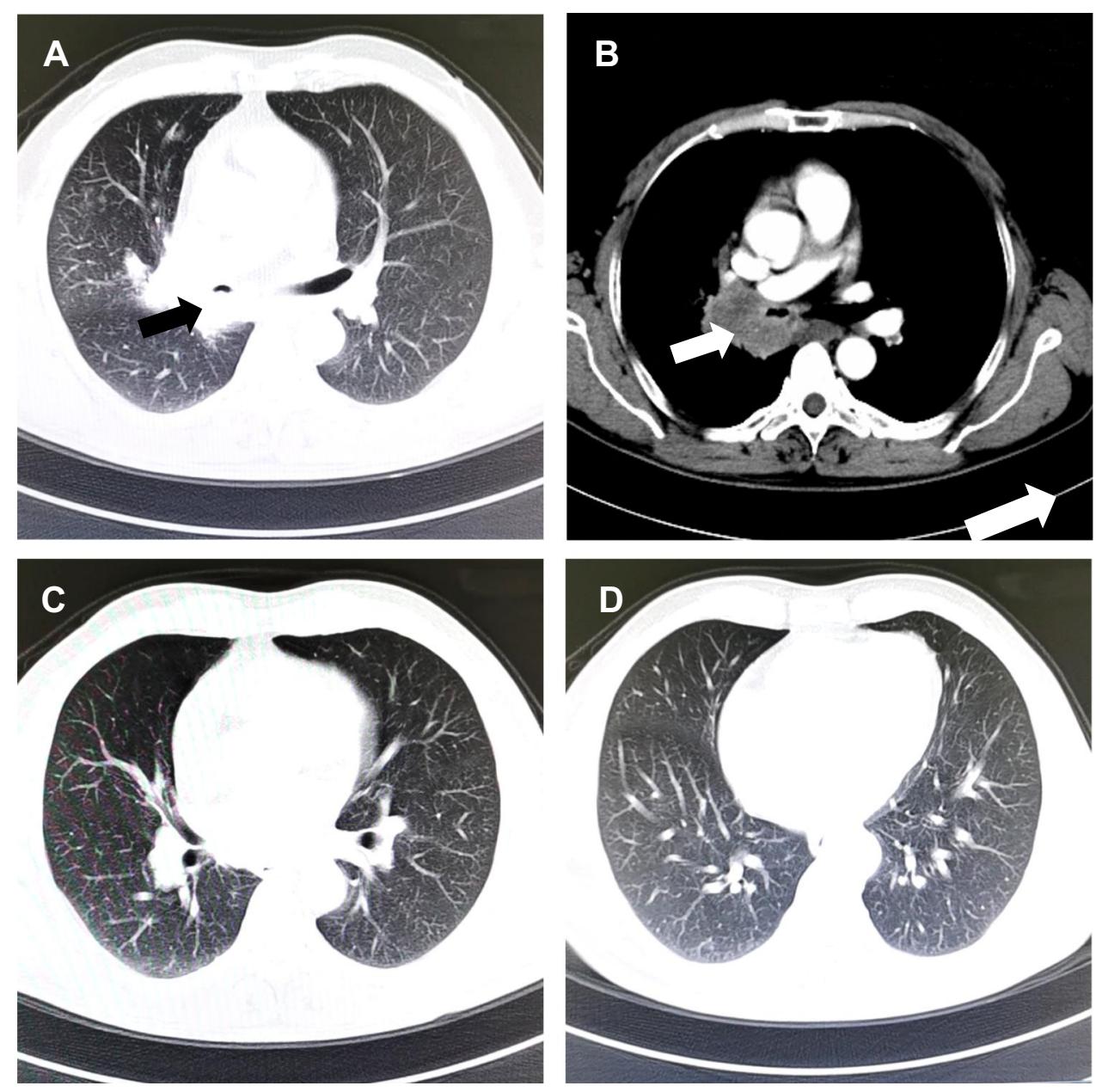

Figure I Chest CT on admission. The lung window of the left upper lobar bronchus level (A), the left lung basal trunk bronchus level (C) and the basal vein level (D). The white arrow indicated the lobular mass with heterogeneous enhancement on mediastinal window in the right hilar region (B). 


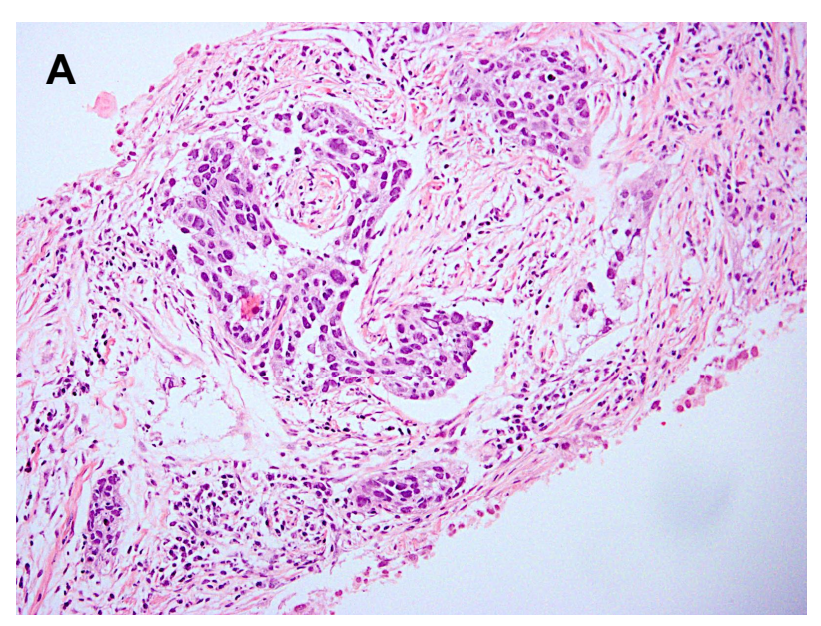

Figure 2 Microscopic findings. The squamous cell carcinoma cells in multiple areas

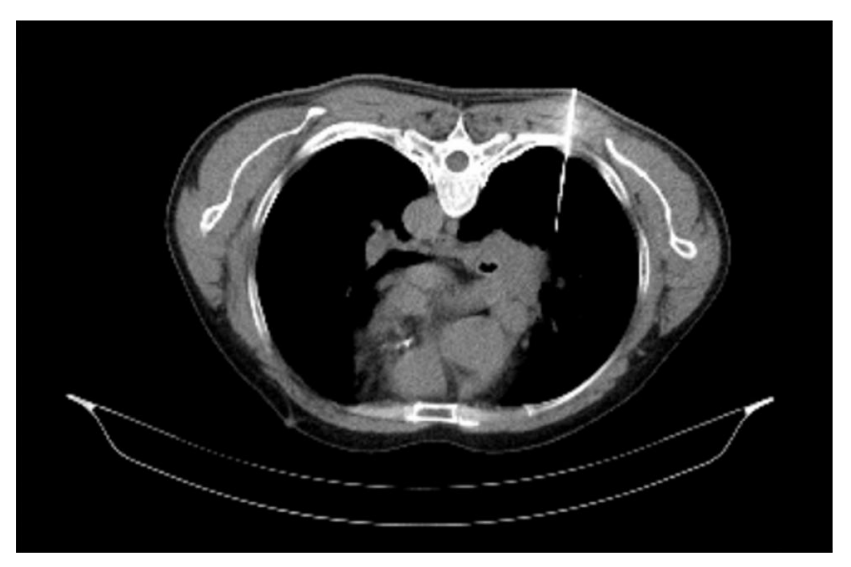

Figure 3 CT-guided percutaneous lung biopsy. The second specimen was obtained at the peripheral site of the mass.

patient was discharged after diagnosed with lung squamous cell carcinoma in consideration of medical expenses and poor prognosis. No more data was collected, and the patient died 6 months later in the telephone return visit.

\section{Discussion}

In the present case, we initially considered the mass in the right hilar region as lung cancer according to the patient's symptoms, radiological features and lesion appearance, which turned out to be TB by TBLB. Further biopsy confirmed the coexistence of TB and squamous cell carcinoma.

Although pathologic results by the TBLB revealed the mass in the right hilar region as TB, a possibility of malignant tumor was considered due to the following reasons. Firstly, the complains of sustaining cough and worsen polypnea indicated an acute progressive disease. Secondly, the mass was located in the right hilar region in our case, as the

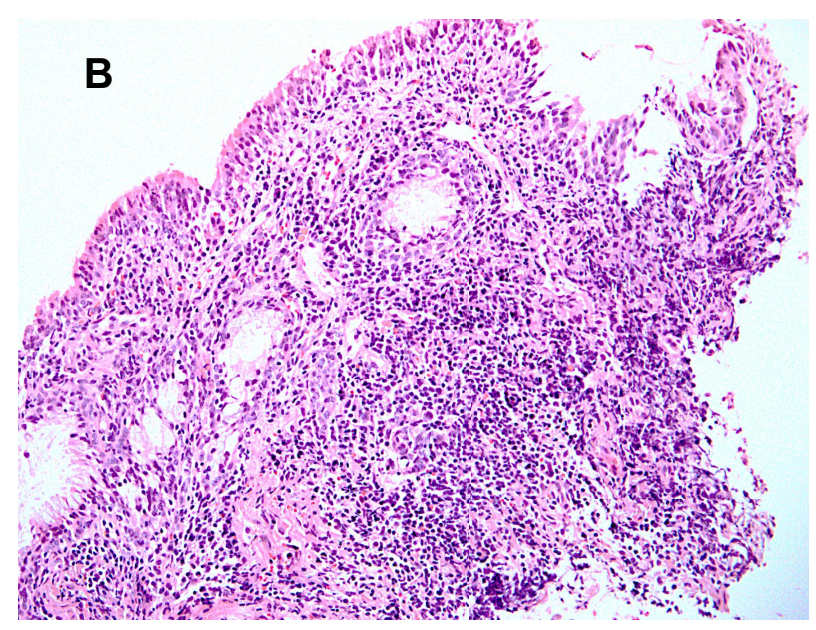

(A) and tuberculous necrosis inflammation (B). (Hematoxylin-eosin stain, x200).

predominant locations of typical TB lesions are often in the superior lobe apicoposterior segment and lower lobe dorsal segment. Also, most benign nodules had smooth margins while our patient had a lobular one. Lastly, the TBLB showed an intuitive invasive nidus which indicated a malignancy. All the evidence showed the suspicion of lung cancer even after the pathological diagnosis of TB, a well-known imitator, was made. So, in consideration of the possibility of coexistence diseases, the CT-guided percutaneous lung biopsy was performed, which finally proved our speculation.

There have been rare reports on the coexistence of lung cancer and pulmonary TB in the same lesion, ${ }^{1-3}$ while more cases occur in the same or different lobes. ${ }^{5,6}$ Ashizawa et al reported a patient with a mass in the left lower lobe undergoing the left lower lobectomy. The specimen showed coexistence of adenocarcinoma and tuberculous granuloma. ${ }^{3}$ Similarly, Byun et al reported a case that a patient, with a mass consisting of cavity and consolidation in the right lower lobe, who underwent a right lower lobectomy. The pathological result in consolidation confirmed an adenocarcinoma and the cavity as TB. ${ }^{2}$ In our case, the CT scan demonstrated a lobular mass in the right hilar region with an irregular margin. And the patient received twice biopsy and diagnosed with TB and squamous cell carcinoma respectively. To our knowledge, here we firstly described a case of lung squamous cell carcinoma and tuberculosis in the same lesion.

More studies noted the relationship between TB and lung cancer while the mechanism remains unknown. ${ }^{4}$ It is difficult to identify the causality between the lung cancer and TB. The lung cancer could arise from a previous pulmonary TB lesion, which is known as a scar theory. Also, the components of TB can cause persistent inflammation response, 
involving in promoting lung cancer development and progression. $^{7}$ In addition, with the progression of lung cancer, the old pulmonary TB could be reactivated. Moreover, cancer-induced immunosuppression and insufficient nutrition can lead the patient to get TB infection easily. ${ }^{8}$ There were more reported adenocarcinoma cases than squamous cell carcinoma. Some authors studied the connection between the adenocarcinoma and TB, displaying the same mutation, ${ }^{9}$ but no significant association was found in squamous cell carcinoma. ${ }^{10}$ In our study, it was more important to find the coexistence of diseases rather than manifest the causality between the lung cancer and the pulmonary TB.

In summary, we reported a case of patient with a lobular mass in the hilar region confirmed as TB and lung squamous cell carcinoma in the same lesion by twice biopsies. This case illuminated that when the mass with cancer-like morphologic features and location instead of typical TB, even the initial pathological result shows TB, coexistence of the diseases should be considered. Further biopsy is recommended to confirm whether there remains the coexistence of lung cancer in the different parts of the same lesion.

\section{Ethical Approval and Consent to Participate}

Informed written consent was obtained from the patient's daughter for publication of this report and any accompanying images. The retrospective study was approved by the institutional review board of West China Hospital of Sichuan University (Number: 2020[390]).

\section{Acknowledgments}

The authors gratefully acknowledge the assistance of Professor Xia Guo at the foreign language college in Sichuan university for her assistance with language modification.

\section{Author Contributions}

All authors contributed to acquisition of data, drafting or revising the article, gave final approval of the version to be published, agreed on the journal to which the article will be submitted and agree to be accountable for all aspects of the work.

\section{Funding}

National Science and Technology Major Project of China, 2018ZX10715-003.

\section{Disclosure}

All the authors have no potential conflicts of interest to disclose.

\section{References}

1. Cicenas S, Vencevicius V. Lung cancer in patients with tuberculosis. World J Surg Oncol. 2007;5:22. doi:10.1186/1477-7819-5-22

2. Byun HG, Yoo JY, Kim SJ, Lee OJ, Yoo MY. Coexistence of lung adenocarcinoma and pulmonary tuberculosis within a single lesion: a rare case report. Medicine (Baltimore). 2019;98:e17378. doi:10.1097/MD.0000000000017378

3. Ashizawa K, Matsuyama N, Okimoto T, et al. Coexistence of lung cancer and tuberculoma in the same lesion: demonstration by high resolution and contrast-enhanced dynamic CT. $\mathrm{Br} J$ Radiol. 2004;77:959-962. doi:10.1259/bjr/31864795

4. Ye MF, Su S, Huang ZH, et al. Efficacy and safety of concurrent anti-tuberculosis treatment and chemotherapy in lung cancer patients with co-existent tuberculosis. Ann Transl Med. 2020;8(18):1143. doi:10.21037/atm-20-5964

5. Zheng L, Yin J, Wang S, et al. Associated factors of co-existent pulmonary tuberculosis and lung cancer: a case-control study. Eur J Clin Invest. 2020;14:e13432.

6. Molina-Romero C, Arrieta $\mathrm{O}$, Hernández-Pando R. Tuberculosis and lung cancer. Salud Publica Mex. 2019;61:286-291. doi:10.21149/ 10090

7. Leung CC. Does tuberculosis increase the risk of lung cancer? Int J Tuberc Lung Dis. 2016;20:712. doi:10.5588/ijtld.16.0265

8. Dutkowska AE, Antczak A. Comorbidities in lung cancer. Pneumonol Alergol Pol. 2016;84:186-192. doi:10.5603/ PiAP.2016.0022

9. Luo $\mathrm{YH}, \mathrm{Wu} \mathrm{CH}, \mathrm{Wu}$ WS, et al. Association between tumor epidermal growth factor receptor mutation and pulmonary tuberculosis in patients with adenocarcinoma of the lungs. $J$ Thorac Oncol. 2012;7:299-305. doi:10.1097/JTO.0b013e31823c588d

10. Liang HY, Li XL, Yu XS, et al. Facts and fiction of the relationship between preexisting tuberculosis and lung cancer risk: a systematic review. Int J Cancer. 2009;125:2936-2944. doi:10.1002/ijc.24636
OncoTargets and Therapy

\section{Publish your work in this journal}

OncoTargets and Therapy is an international, peer-reviewed, open access journal focusing on the pathological basis of all cancers, potential targets for therapy and treatment protocols employed to improve the management of cancer patients. The journal also focuses on the impact of management programs and new therapeutic agents and protocols on patient perspectives such as quality of life, adherence and satisfaction. The manuscript management system is completely online and includes a very quick and fair peer-review system, which is all easy to use. Visit http://www.dovepress.com/ testimonials.php to read real quotes from published authors. 